\title{
De la monoparentalidad a la reconstitución familiar: un análisis a partir de fuentes transversales
}

\author{
Rocío Treviño \\ Jordi Gumà \\ Universitat Autònoma de Barcelona. Centre d'Estudis Demogràfics \\ rtrevino@ced.uab.es; jguma@ced.uab.es
}

\section{Resumen}

El artículo estudia qué perfil sociodemográfico de las madres monoparentales se asocia con la reconstitución familiar a partir de fuentes estadísticas transversales: ¿ ¿Son diferentes las mujeres que están en monoparentalidad de las mujeres que están en un núcleo reconstituido aportando ellas a los hijos?

En primer lugar, se examina la coherencia de las fuentes en la descripción del perfil sociodemográfico de las madres de núcleos monoparentales y reconstituidos. En segundo lugar, se analizan las variables sociodemográficas de las mujeres asociadas a la reconstitución familiar desde una situación de monoparentalidad mediante un análisis multivariable por regresión logística. Se utilizan el censo de población de 2001 y las Encuestas de Población Activa de 1999 a 2010. Los resultados muestran una gran coherencia de las fuentes estadísticas y señalan que no son las variables de status individual (ocupación y nivel de instrucción de las mujeres) las que mejor explican las diferentes propensiones a la reconstitución familiar de las madres monoparentales.

Palabras clave: familias reconstituidas; familias monoparentales; familia; hogar.

Abstract. From Single-Parenting to Family Reconstitution: An Analysis Based on CrossSectional Data Sources

This article aims to identify which sociodemographic profile of single-parenting mothers is associated with family reconstitution. Are single-parenting women different from women in a reconstituted nucleus?

After examining the coherency of statistical sources describing the sociodemographic profile of mothers in single-parent and reconstituted nuclei, the article analyses the soci-

* Este trabajo debe considerarse un producto del proyecto «Hogares de familias reconstituidas y equidad de género: ¿nuevas paradojas?” Plan Nacional de I+D+I -2008-2011) ref. CSO200800654 
odemographic variables of former single-parenting women now associated with family reconstitution using multivariate logistic regression analysis. The Population Census of 2001 is used along with Labour Force Survey from 1999 to 2010. The results show a high degree of consistency in the statistical sources and indicate that individual status variables (occupation and educational level of women) are not those that best explain the different propensities towards family reconstitution among single-parenting mothers.

Keywords: stepfamily; one-parent family; family; household.
Sumario
1. Introducción
4. Resultados
2. Estado de la cuestión
5. Reflexiones finales
3. Métodos y fuentes
Referencias bibliográficas

\section{Introducción}

El conocimiento sociológico que en España se tiene de las familias reconstituidas, aquéllas que incluyen una pareja unida con al menos un hijo no común, es muy precario y está muy por debajo de la demanda de información que, sobre todo, desde los medios de comunicación y desde las distintas administraciones públicas, se exige en relación a los aspectos más novedosos del cambio familiar reciente: el divorcio, las parejas de doble ingreso, las familias monoparentales, las familias reconstituidas, las parejas infecundas, etc. No obstante, la proliferación de estudios sobre estas familias desde disciplinas tan dispares como la psiquiatría, la psicología, el derecho o la antropología sugiere tanto la relevancia social del fenómeno, como el interés académico del mismo (García-Mina et al., 2003; Castanys, 2006; Roigé Ventura, 2006; Guilarte Martín-Calero, 2009).

Desde la sociología, las razones de esta laguna en el conocimiento de las familias reconstituidas son de naturaleza diversa, pero destacan dos. En primer lugar, la limitación de las fuentes estadísticas disponibles para su estudio y, como ejemplo paradigmático, se destacará que la fuente estadística por antonomasia para estudiar el perfil sociodemográfico transversal de los hogares desde una perspectiva cuantitativa, el censo de población, no permite reconstruir este tipo de familia o de núcleo familiar hasta el año 2001. Esto ha determinado la utilización de metodologías cualitativas en su estudio (Roigé Ventura, 2006; Rivas Rivas, 2008; Solsona, 2011) y que, en los trabajos en los que se analizan específicamente los cambios estructurales de las familias desde perspectivas cuantitativas (Flaquer y Soler, 1990; Solsona y Treviño, 1990; Valero, 1995; Requena, 1999; Garrido et al., 2000; Jurado, 2005), no se aborden los núcleos reconstituidos.

Pero otra razón de peso de la falta de estudios cuantitativos de las familias reconstituidas es que, detrás de un núcleo familiar reconstituido, hay una 
pluralidad de perfiles sociodemográficos en función de la diversidad de trayectorias familiares que han dado lugar a la reconstitución de tal núcleo. Este hecho exige la distinción analítica de diferentes tipos de reconstitución y, en un contexto de fuerte limitación de fuentes estadísticas, dificulta su estudio. Así, Treviño et al. (en prensa) señalan la heterogeneidad sociodemográfica de los núcleos reconstituidos en España en función de diversos aspectos: 1) si los hijos no comunes de la pareja son aportados a la convivencia por el hombre, por la mujer o por ambos; 2) si existen también hijos comunes en el hogar, 3) o si los lazos de unión son matrimoniales o consensuales. Esto, unido a que se trata de un fenómeno emergente en nuestro panorama familiar (y nos estamos refiriendo, eso sí, a la reconstitución tras un episodio de divorcio o separación), se junta con la necesidad de disponer de encuestas de tamaño muestral lo suficientemente grandes como para asegurar la representatividad estadística de los análisis realizados. En ausencia de muestras específicas y recientes que permitan abordar con generosidad el perfil de los núcleos familiares reconstituidos, se ha optado por analizarlo enlazando los microdatos de las encuestas de población activa (EPAS) en diferentes períodos, sin dejar de mostrar la coherencia o la incoherencia de sus resultados con los derivados de la explotación de los microdatos del último censo de población.

Fuera de nuestras fronteras, el uso de encuestas de tipo biográfico se ha impuesto como la vía más pertinente para analizar la heterogeneidad de los perfiles sociodemográficos de la monoparentalidad a través del estudio de las trayectorias familiares que dan lugar a la formación de los núcleos reconstituidos. Para unos, formar parte de una familia reconstituida puede ser la primera experiencia de paternidad, de maternidad o de vida conyugal; para otros y, sobre todo, otras, la reconstitución está precedida de un periodo de monoparentalidad (Juby et al., 2001), pero, en nuestro país, las encuestas biográficas son una asignatura pendiente y, de momento, no se puede recurrir a ellas para un estudio dinámico de la reconstitución ${ }^{1}$.

En este trabajo, nos proponemos abordar la cuestión de si existe un determinado perfil sociodemográfico en las mujeres que forman un núcleo reconstituido después de un episodio de monoparentalidad. Pero, dado que no contamos con datos biográficos para abordar esta materia que, en esencia, exigiría una perspectiva de análisis dinámico, se han seleccionado, utilizando fuentes transversales, a las mujeres que alguna vez han experimentado la monoparentalidad (las mujeres que en el momento del análisis están en monoparentalidad y las mujeres que forman un núcleo reconstituido en el cual aportan ellas los

1. La última encuesta de fecundidad, la Encuesta de fecundidad, familia y valores (CIS), que es la primera de la saga que recoge información sobre con quien se quedan los hijos después de una ruptura, tiene un tamaño muestral que no resiste el análisis de la reconstitución familiar después de una ruptura. Además, sólo está centrada en mujeres y no recoge, en el historial femenino de unión, si los cónyuges respectivos cuentan o no con hijos. En la reciente encuesta biográfica realizada en Cataluña con un tamaño muestral de cerca de 27.000 individuos, Enquesta demográfica de Catalunya 2007, tampoco se recoge en el historial de parejas ninguna de estas dos informaciones. 
hijos no comunes a la convivencia con la pareja) para estudiar qué perfil sociodemográfico se asocia con la reconstitución familiar. ¿ Son diferentes las mujeres que están en monoparentalidad de las mujeres que están en un núcleo reconstituido en el que aportan ellas a los hijos? No hablaremos en términos de causalidad, pero sí de asociación. De este modo, pretendemos aproximarnos, con una metodología transversal, al conocimiento de las características sociodemográficas de las mujeres monoparentales asociadas a la reconstitución familiar. La oportunidad de restringir el análisis a los núcleos reconstituidos en los que los hijos no comunes de la pareja son aportados por la mujer, se justifica porque éstos constituyen el $88 \%$ del total de núcleos reconstituidos con algún menor de 29 años, según datos de la EPA del segundo trimestre de 2010. Y dado que la cantera o el universo que está a riesgo de formar un núcleo reconstituido, en el que son las mujeres las que aportan a los hijos no comunes, la constituye los núcleos monoparentales femeninos, necesariamente éstos tienen que ser el referente de nuestro análisis, es decir, del perfil que se asocia a la reconstitución después de un supuesto episodio de monoparentalidad. No hay que olvidar que la salida de mujeres de una situación de monoparentalidad a causa de una unión implica la entrada o la formación de un núcleo reconstituido en que ellas aportan al hogar a los hijos no comunes ${ }^{2}$ del núcleo reconstituido.

\section{Estado de la cuestión}

El análisis de los determinantes de la reconstitución familiar después de un episodio de monoparentalidad ha estado ligado a los estudios focalizados en la pobreza de las familias monoparentales, principalmente realizados desde el campo de la política social. Desde el momento en que se ha constatado que los núcleos monoparentales están asociados a un mayor riesgo de pobreza (Chambaz, 2001), la reconstitución familiar a través de la unión ha sido analizada como un posible mecanismo reductor de la pobreza en las familias monoparentales (Le Bourdais et al., 1995; Rendall, 1999; Edin, 2000). De aquí se derivaría que las mujeres que se encuentran en un hogar reconstituido tendrían un perfil sociodemográfico de más bajo estatus que sus homólogas monoparentales, ya que la reconstitución seleccionaría a las más necesitadas de una pareja para que ésta las ayude económicamente.

En el caso de España, Treviño (2011) destaca, en línea con la hipótesis anterior, que en las cohortes nacidas entre 1941 y 1960, analizadas en 1991, la reconstitución familiar por unión selecciona a las mujeres monoparentales de menor educación y de menor participación laboral.

Algunas otras pistas de cuáles pueden ser las variables sociodemográficas asociadas a la reconstitución desde una situación de monoparentalidad también pueden encontrarse en los estudios sobre las pautas de formación de la pareja

2. El porcentaje de núcleos reconstituidos en los que existen hijos no comunes de ambos miembros de la pareja constituye sólo un $2 \%$ del total de núcleos reconstituidos con algún menor de 29 años. 
en segundas uniones (Bumpass et al., 1990; Coleman et al., 2000; De Graaf y Kalmijn, 2003), pero los trabajos que abordan específicamente los rasgos de las madres monoparentales que las hace más susceptibles de reconstitución familiar o que se centran en las características sociodemográficas específicas de las madres monoparentales y reconstituidas son muy escasos, si bien ahondan en considerar como variables de estudio, la edad de las mujeres y el capital humano, léase, la situación de empleo y el nivel educativo, así como la carga reproductiva de las mujeres (McCarthy y Cherlin, 1990; Desrosiers y Le Bourdais, 1993; Ermisch y Wrigth, 1991; Juby et al., 2001).

Los resultados en relación con el efecto negativo de la edad en las probabilidades que tienen las mujeres de volverse a casar o de formar un núcleo familiar reconstituido son concluyentes: la edad muestra una relación inversa. Los resultados en relación con el efecto del nivel educativo de las mujeres o de la situación de actividad en la propensión a volverse a unir no son, en cambio, tan claros. En referencia a la ocupación, por ejemplo, algunos han encontrado una relación positiva entre reconstitución familiar por unión y ocupación (Ermisch y Wrigth, 1991). Esta relación se ha argumentado alegando que, probablemente, la ocupación facilita el encontrar otra pareja, al ampliar el ámbito de las relaciones sociales. Así, Le Bourdais et al. (1995) señala que sólo se detecta esta relación positiva cuando se utiliza el indicador de ocupación en el momento de entrada en monoparentalidad de las mujeres y no cuando se tiene presente el efecto de la ocupación a lo largo de todo el episodio de monoparentalidad.

El efecto del número y la edad de los hijos tampoco está claro en la bibliografía, aun presuponiéndose teóricamente que la carga emocional, financiera y de tiempo de las madres monoparentales varía en función del número y la edad de los hijos, y que esta carga diferencial va a impactar en las probabilidades de unión. Le Bourdais et al. (1995) sugieren que la confusión de resultados en relación con el impacto de esta variable en la reconstitución deriva de la pluralidad de métodos utilizados para abordar esta cuestión. Algunos estudios señalan que el número de hijos tiene poco impacto en las probabilidades de reconstitución familiar o que sólo un número muy grande de hijos desincentiva la unión entre las madres monoparentales; otros apuntan que la propensión a las segundas nupcias es menor a medida que aumenta el número de hijos dependientes entre las mujeres, o que lo que cambia la suerte de segundas nupcias es tener o no tener hijos (Ermisch y Wrigth, 1991; Mott y Moore, 1983). Le Bourdais et al. (1995), en cambio, sostienen que, cuando se trata de las expectativas del marido, el número de hijos incrementa las probabilidades de volverse a casar.

\section{Métodos y fuentes}

Los resultados presentados derivan de una explotación de los microdatos de las encuestas de población activa (EPA) de 1999 a 2010. Dado que la muestra de mujeres en núcleos reconstituidos en los cuales ellas aportan a los hijos no comunes a la convivencia, es decir, la muestra de mujeres que están en recons- 
titución familiar habiendo pasado forzosamente por una situación de monoparentalidad, es muy pequeña, se ha procedido a agrupar las EPAs para el análisis. La agrupación se ha realizado en dos periodos, 1999-2004 y 2005-2010, a partir de la primera EPA, lo cual permite estudiar los núcleos reconstituidos juntando los semestres, teniendo en cuenta que cada trimestre se renueva $1 / 6$ de la muestra. Para el primer periodo, disponemos de una muestra de 20.322 mujeres que encabezan núcleos monoparentales y de 2.740 mujeres que forman núcleos reconstituidos en los cuales ellas aportan a los hijos no comunes. Para el período 2005-2010, la muestra respectiva es de 21.204 monoparentales y 3.858 mujeres en reconstitución.

No estando todavía disponibles los datos del censo de 2011, se han usado también los microdatos del censo de población de 2001 en aras de comprobar la solidez de los resultados de la EPA. Hay que tener presente que esta fuente, como la mayoría de encuestas, presenta ciertos sesgos en la captación de determinados tipos de hogar (Garrido et al., 2000)3. Para la identificación de los núcleos, se han seguido dos criterios: 1) la relación entre la madre o el padre con el hijo o la hija tiene preferencia sobre la del hijo o la hija con la madre o el padre; y la relación de pareja siempre tiene preferencia sobre la relación de la madre o el padre con el hijo o la hija. Los núcleos reconstituidos se identifican cuando existe un individuo en posición de hijo (sin pareja y sin hijos) cuya madre convive con un hombre que no es su padre. Se han seleccionado exclusivamente los núcleos monoparentales femeninos y los reconstituidos en los que sólo las mujeres aportan a la convivencia a los hijos no comunes, considerando solo los núcleos con algún hijo menor de 29 años.

Se han utilizado, asimismo de forma puntual, los microdatos de la Enquesta demogràfica 2007 (ED) realizada en Cataluña para ilustrar alguna cuestión específica. Dicha encuesta actualiza y da seguimiento a la información procedente de los censos y de los padrones de población. Aunque no abarca el ámbito estatal, dada la escasez de fuentes estadísticas para estudiar los núcleos reconstituidos, la generosidad de su muestra (de 27.000 individuos que permiten contar con una submuestra de 257 núcleos reconstituidos de madre que aporta hijos) y el hecho de que las nuevas familias presentan en Cataluña un perfil sociodemográfico comparable al del conjunto del Estado (Treviño et al., en prensa), ha resultado un referente útil para analizar la discrepancia puntual en el perfil de una variable mostrado por el censo y la EPA en la descripción de las características sociodemográficas de las madres monoparentales y reconstituidas.

Para el análisis multivariable, se han seleccionado las mujeres que alguna vez han estado en monoparentalidad, es decir, aquéllas que, en el periodo

3. La mayoría de las encuestas no realizan substituciones selectivas según la estructura de los hogares de la muestra teórica que, por la razón que sea, no resultan encuestables. Esto provoca ciertos sesgos en la muestra efectiva de los hogares según la estructura de los mismos. De este modo, determinados tipos de hogares, dependiendo de las características de sus miembros, resultan subestimados. Así ocurre, por ejemplo, con los hogares de parejas biactivas o con los hogares unipersonales cuyo único miembro está ocupado y resulta más difícil hallarlo en su casa. 
estudiado, encabezaban un núcleo monoparental y aquéllas que formaban un núcleo reconstituido con sus hijos. Se han estudiado las variables asociadas a la reconstitución familiar mediante un modelo de regresión logística en el que la variable dependiente es dicotómica y señala si se está o no en un núcleo reconstituido. Los resultados de las regresiones se presentan en forma de oddsratios (los $\exp (b)$ de la regresión) o "propensiones relativas»: si la probabilidad es $\operatorname{Pr}$, la propensión es $\operatorname{Pn}=\operatorname{Pr} /(1-\operatorname{Pr})$ y la propensión relativa es $\operatorname{Pn}(a) / \operatorname{Pn}(b)$, donde $a$ representa la categoría que toma una variable y $b$, la categoría de la variable que sirve de referencia. La ventaja de utilizar la regresión estriba en que se estandariza la propensión del fenómeno analizado según las diferentes variables que se incluyen en el modelo, pues se tiene en cuenta el efecto del conjunto de las variables independientes introducidas en el mismo sobre la variable dependiente y, además, proporciona los niveles de significación estadística de esta propensión.

\section{Resultados}

\section{A. Estimaciones del fenómeno y evolución}

En el gráfico 1, se presenta la clasificación de los núcleos reconstituidos por tipos, en función de si los hijos no comunes del núcleo los aporta el varón de la pareja o la mujer (columnas verticales) y de si el núcleo reconstituido lo conforman además hijos comunes de la pareja (columnas horizontales). La reconstitución familiar objeto de estudio corresponde a la de los núcleos reconstituidos en los que las mujeres aportan ellas a los hijos y, por tanto, la reconstitución deviene tras un episodio femenino de monoparentalidad. Tal y como puede apreciarse en el gráfico, éstos constituyen la mayoría de ellos (el $88 \%$ del total). Como la formación de un núcleo reconstituido se produce la mayor parte de las veces tras un episodio de separación (legal o de hecho) o tras un divorcio, y como la custodia de los hijos recae mayoritariamente en la mujer, dicho dato no es nada sorprendente. Esta preponderancia se produce a pesar de los cambios que, en relación con la custodia, introduce la reforma de la Ley de divorcio de 2005, la denominada "Ley de divorcio exprés», que establece la custodia compartida como preferente si no hay acuerdo entre los cónyuges. Efectivamente, en un $84 \%$ de los divorcios y separaciones registrados en el año 2009, la custodia recayó en la madre y sólo el 6\%, en el padre. Por separado, en el $6 \%$ de las separaciones la custodia fue compartida, mientras que en los divorcios ésta representó un $10 \%$ según datos del INE ${ }^{4}$.

Un dato que causa mayor sorpresa es la «solidez estructural» que tienen las familias reconstituidas en España, si nos atenemos al elevado peso que asumen los núcleos reconstituidos donde también conviven hijos comunes de la pareja en el total de núcleos reconstituidos. Entre los núcleos de parejas reconstituidas en los que las mujeres aportan a los hijos no comunes, en un

4. Según datos de la estadística de nulidades, separaciones y divorcios de 2009. 
Gráfico 1. Distribución de los núcleos reconstituidos por tipo (absolutos y en porcentaje sobre el total). 2010

Total núcleos o familias reconstruidas: 221.000

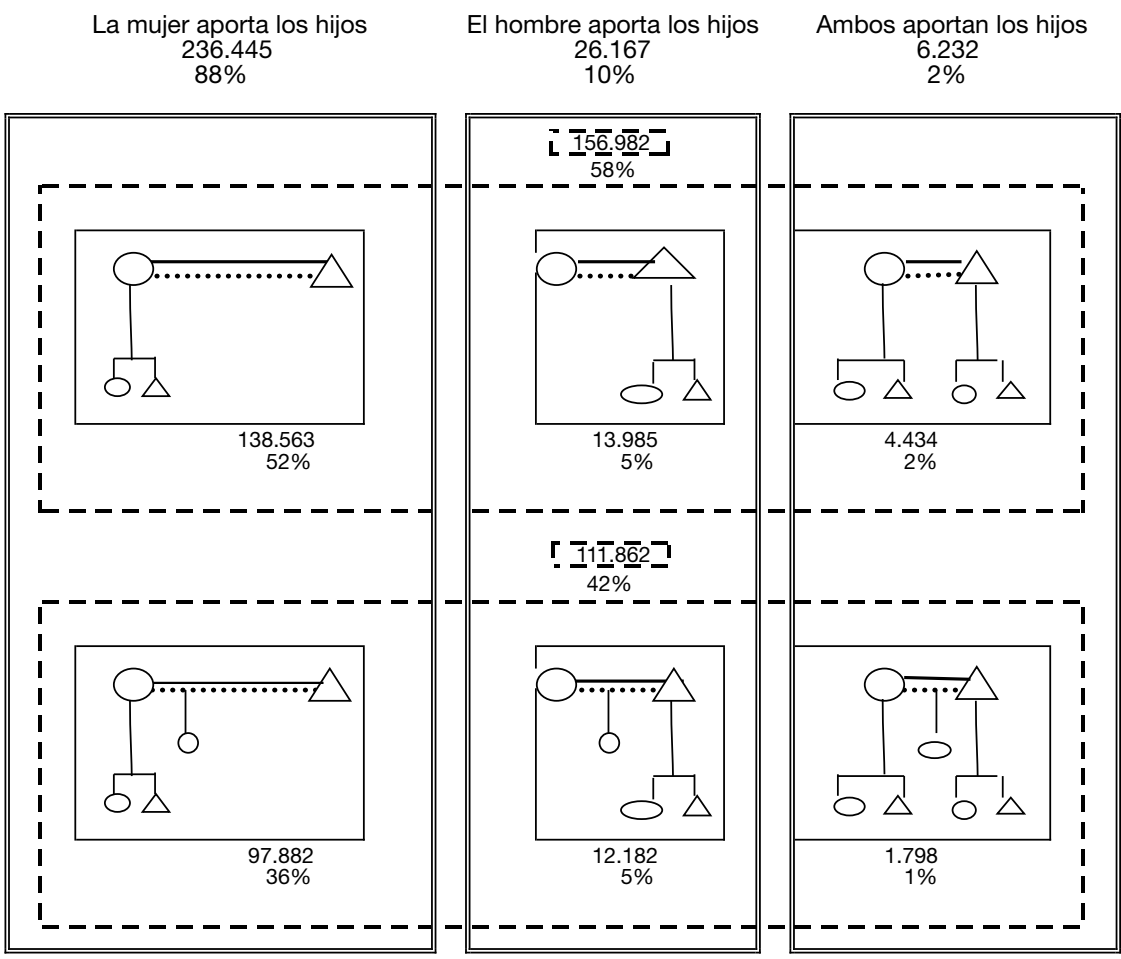

Nota: los seis tipos de núcleos reconstituidos se multiplican por 2 si se tiene en cuenta si la unión de la pareja es matrimonial (en línea continua) o consensual (en línea discontinua)

La línea punteada multiplicaría los tipos por 2 si se tuviese en cuenta si la unión es consensual o no. Núcleos de parejas heterosexuales con hijos menores de 29 años.

Fuente: elaboración propia a partir de los microdatos de la EPA (II trimestre).

$41 \%$ también hay hijos comunes y este peso aún es más elevado en los escasos núcleos reconstituidos en los que los hombres conviven con sus propios hijos: un $47 \%$. Algunos estudios han mostrado que la llegada de un hijo común transforma la naturaleza del núcleo familiar reconstituido, al crear un vínculo genético entre todos los miembros que la componen (Juby et al., 2001). El hijo común actuaría como un mecanismo reforzador de la institución de la pareja, puesto que dotaría de mayor estabilidad a la misma.

Dado que la cantera de la reconstitución familiar a analizar la constituyen las madres en situación de monoparentalidad, en el gráfico 2 se muestra la evolución alcista de estos núcleos sobre el total de familias monoparentales con hijos menores de 29 años. En el año 2010, los núcleos monoparentales encabezados por mujeres, cerca de un millón de núcleos, constituyen el 13\% del 
Gráfico 2. Porcentajes de núcleos monoparentales femeninos y de núcleos reconstituidos en los que es la mujer la que aporta a los hijos (años 1999-2010)

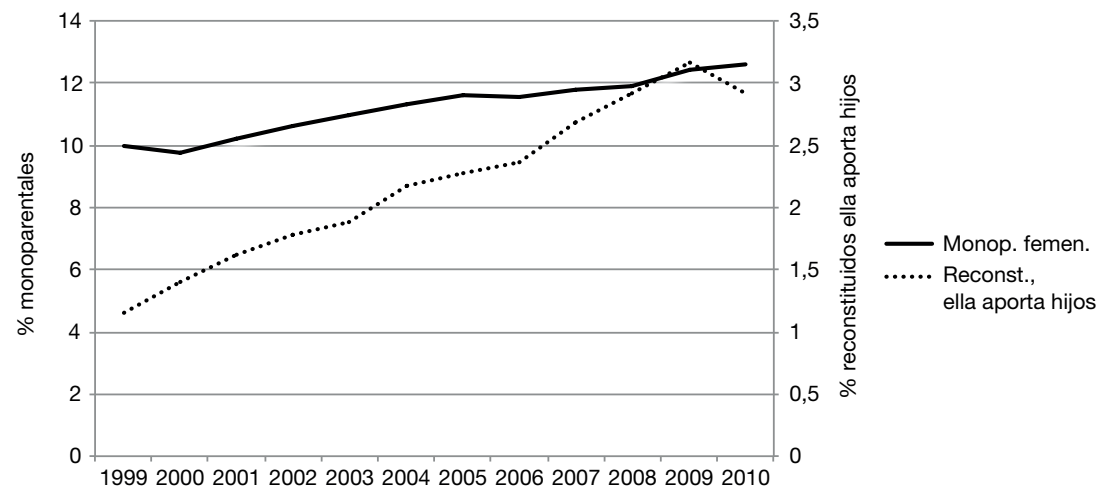

Núcleos con hijos menores de 29 años.

Fuente: elaboración propia a partir de los microdatos de la EPA (II trimestre).

total de familias con hijos menores de 29 años y los reconstituidos en los que las mujeres aportan a los hijos, cerca de 240.000 núcleos, el 3\% de este total.

La evolución de la intensidad de la reconstitución familiar con datos transversales se mide relacionando los núcleos reconstituidos con la población susceptible de reconstitución, en nuestro caso, con los núcleos monoparentales femeninos. La evolución es también claramente alcista. En 1999, los núcleos reconstituidos en los que la mujer aporta a los hijos se cifraban en 11 por 100 núcleos monoparentales femeninos y, en el año 2010, la relación alcanza a 25 reconstituidas por 100 monoparentales. El número de estos núcleos reconstituidos no sólo depende del volumen de las entradas en situación de monoparentalidad, sino también de la velocidad de las salidas por unión, que es lo mismo que las entradas en situación de reconstitución. El indicador señala un incremento de la importancia de la reconstitución en relación con la monoparentalidad.

\section{B. Los perfiles diferenciados de las mujeres monoparentales y de las mujeres reconstituidas: análisis descriptivo}

\section{La edad}

Para el análisis comparativo del perfil sociodemográfico de las mujeres en núcleos monoparentales y de las que forman un núcleo reconstituido, se han agrupado las EPAs en dos periodos, 1999-2004 y 2005-2010, con el fin de evitar las limitaciones del tamaño de la muestra de núcleos reconstituidos en la encuesta. Se presentan, asimismo, los resultados comparativos realizados en función de los datos del censo de población de 1991, con el propósito de verificar la solidez de la encuesta. Las mujeres que se encuentran en núcleos reconstituidos tienen un perfil de edad mucho más joven que las mujeres de núcleos monoparentales (ver gráfico 3) y esto es así a pesar de que el episodio 
Gráfico 3. Distribución por edad de los núcleos reconstituidos en los que ella aporta a los hijos comunes y de los núcleos monoparentales femeninos (años 1999-2004 y 2005-2010)

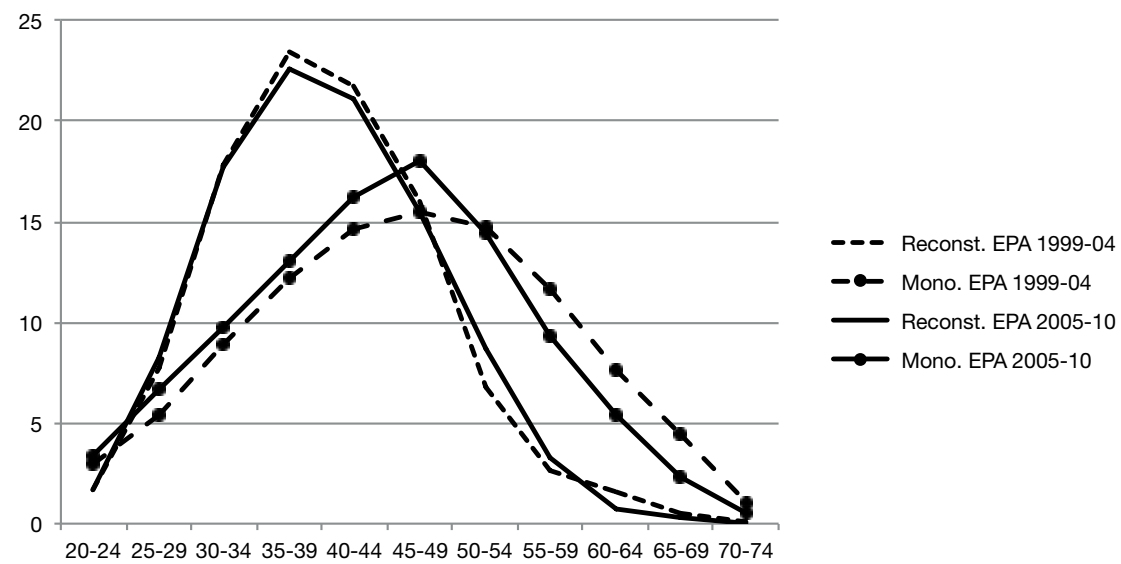

Núcleos con hijos menores de 29 años.

Fuente: elaboración propia a partir de los microdatos de la EPA.

de reconstitución es posterior al de monoparentalidad. Dicho dato refuerza los resultados señalados en la bibliografía de que la reconstitución selecciona a las mujeres más jóvenes.

La evolución seguida de un periodo a otro no marca apenas diferencias en el patrón de distribución por edad de las mujeres de los núcleos reconstituidos, pero sí de los monoparentales, que presenta un perfil de edad más rejuvenecido en el último período. Este rejuvenecimiento del perfil transversal de la monoparentalidad podría deberse a un cambio de calendario del fenómeno, pero también a un cambio generacional o a ambos efectos a la vez. El hecho de que contemos con grupos generacionales muy amplios y sólo dos momentos de observación impide dar una respuesta. Dado que se está observando a grupos de generaciones con un constante atraso en la formación familiar y que los datos que proporciona el INE sobre divorcios y separaciones por duración de matrimonio ${ }^{5}$ no señalan cambios significativos de calendario, este rejuvenecimiento pudiera ser efecto del incremento de las entradas en situación de monoparentalidad de las mujeres de los grupos de edad intermedios. Estos perfiles diferenciados por edad de las madres monoparentales y reconstituidas se confirman con los datos censales del año 2001.

\section{El número de hijos}

Las mujeres de núcleos reconstituidos no sólo tienen un perfil más joven en edad que sus homólogas monoparentales, sino también mayor número de hijos (ver gráfico 4). Los núcleos de un sólo hijo constituyen casi la mitad

5. Aunque la estadística de nulidades, separaciones y divorcios permite al respecto todavía una serie de corto alcance. 
Gráfico 4. Distribución de los núcleos monoparentales femeninos y de los reconstituidos en los que ella aporta a los hijos no comunes. Datos agrupados por número de hijos (comunes y sólo de la madre) (fechas y fuentes diversas)

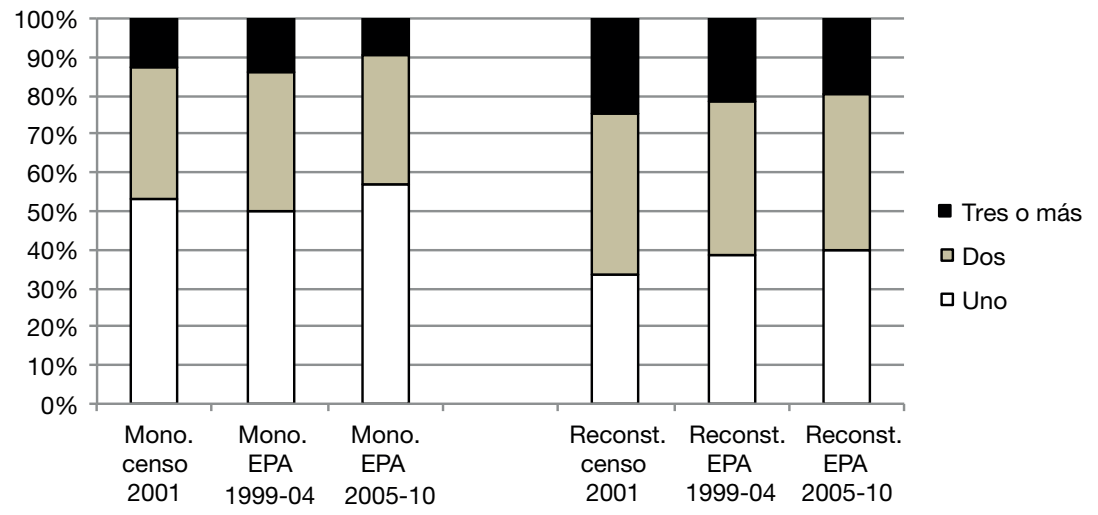

Núcleos con hijos menores de 29 años.

Fuente: elaboración propia a partir de los microdatos del censo y de la EPA.

de los núcleos monoparentales femeninos; mientras que entre los núcleos en los que la mujer aporta a los hijos comunes, éstos no sobrepasan en ningún periodo el $40 \%$. En cambio, los de tres y más hijos no sobrepasan apenas el $10 \%$ en el primer caso y en el segundo suponen cerca del $20 \%$. No obstante, tanto en los núcleos de las madres de núcleos monoparentales como en los de reconstituidos, ha aumentado, entre un período y otro, el peso de los núcleos más pequeños en paralelo al conocido descenso de la fecundidad del conjunto de los núcleos.

Ahora bien, tal como muestra el gráfico 5, esta proporción más elevada de núcleos de mayor tamaño entre las madres de familias reconstituidas que entre las monoparentales se explica por la fecundidad de los primeros. Como se puede ver en parte de la bibliografía foránea (Prskawetz et al., 2003), y aunque aquí sólo dispongamos de una visión transversal del fenómeno, la formación de una segunda unión alienta la fecundidad, ya que suele producirse en el período reproductivo de las mujeres. Se observa que, si bien hay un claro retroceso de altos órdenes de paridad en el conjunto de uniones, la constitución de una segunda unión tiene un efecto de "compromiso" que alienta la fecundidad en la nueva pareja. El porcentaje de núcleos reconstituidos con hijos de ambos miembros de la pareja es relativamente elevado en el conjunto de núcleos reconstituidos, pero es más alto cuanto más reducido es el número de hijos no comunes. Así, según datos censales, en un 53\% de los núcleos reconstituidos en los que la madre aporta a un solo hijo no común, hay también hijos de ambos miembros de la pareja; en los núcleos reconstituidos en los que se convive con dos hijos de la madre, el porcentaje de los que «sellan» la unión con un hijo común se rebaja a un $38 \%$. Indudablemente, cabe dar cuenta de un posible sesgo de diferencias de composición por edad de las madres en la explicación de este último patrón. 
Gráfico 5. Distribución de los núcleos reconstituidos en los que ella aporta a los hijos no comunes por número de hijos sólo de la madre (diversas fechas y fuentes)

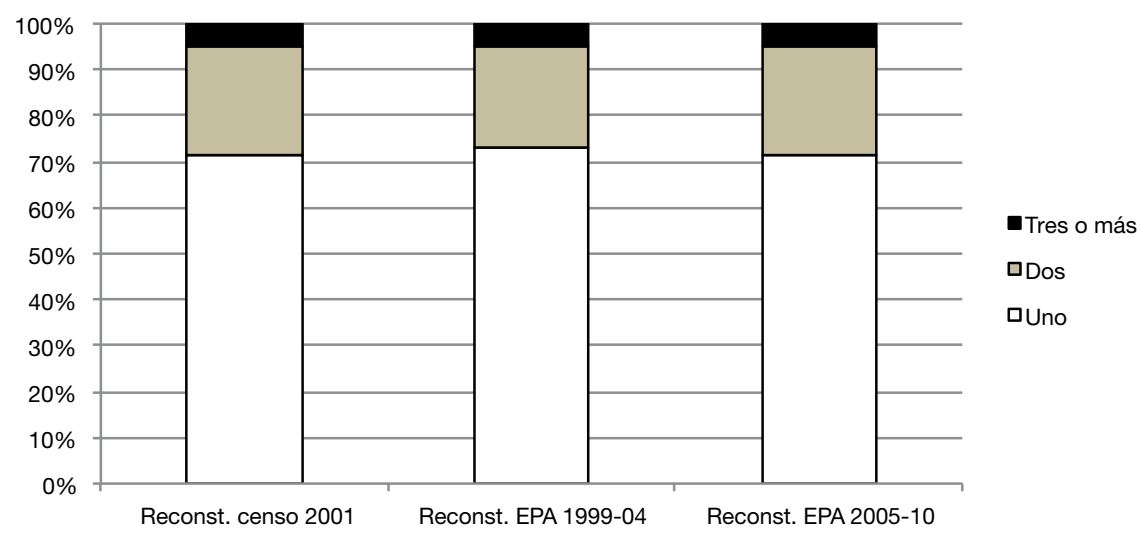

Núcleos con hijos menores de 29 años.

Fuente: elaboración propia a partir de los microdatos del censo y de la EPA.

Gráfico 6. Distribución de los núcleos reconstituidos y de los monoparentales femeninos en los que ella aporta a los hijos no comunes. Datos basados en la edad del hijo menor del núcleo (diversas fuentes y períodos)

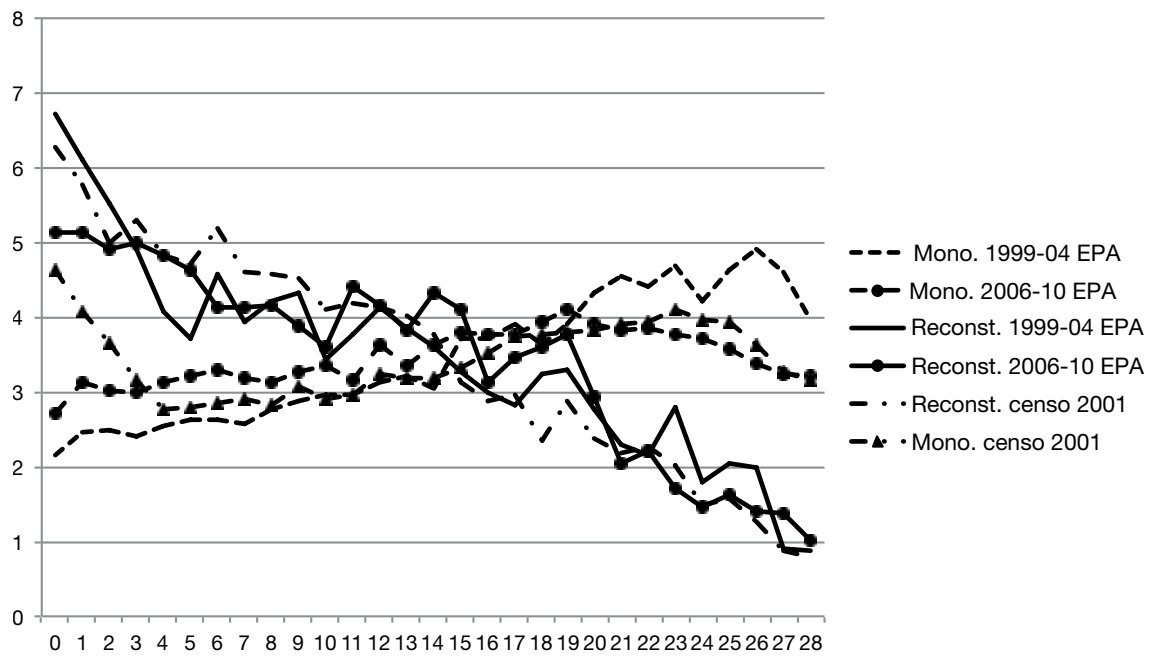

Núcleos con hijos menores de 29 años.

Fuente: elaboración propia a partir de los microdatos del censo y de la EPA.

\section{La edad de los hijos}

El perfil más joven por edad de las madres reconstituidas y el hecho de que la reconstitución conlleve una fecundidad añadida explica la mayor proporción de núcleos cuyos hijos menores son de corta edad entre estas madres frente a las monoparentales (ver gráfico 6). Esta diferencia en la edad de los hijos 
Gráfico 7. Distribución de las madres monoparentales y de las de núcleos reconstituidos en los que ella aporta a los hijos no comunes por estado civil (diversas fuentes y períodos)

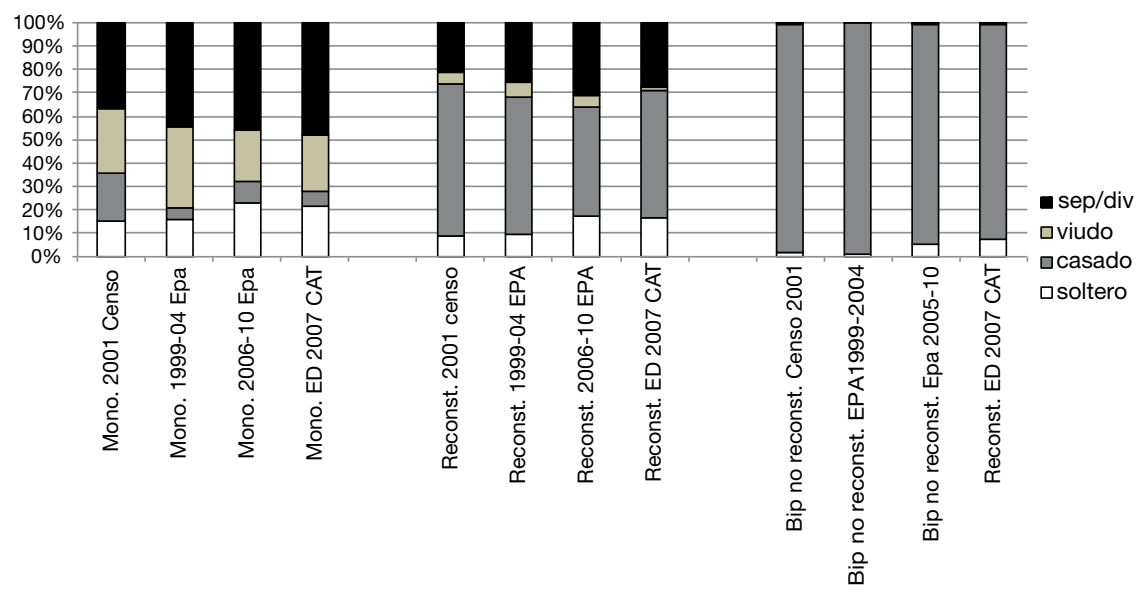

Núcleos con hijos menores de 29 años.

Fuente: elaboración propia a partir de los microdatos del censo, de la EPA y de la Enquesta demográfica 2007 realizada en Cataluña.

no cambia en los dos periodos considerados y es coherente con el patrón que dibuja el censo de población.

\section{El estado civil de las madres}

Ante la escasez de encuestas biográficas, el estado civil se perfila como variable indirecta de la trayectoria que puede esconderse tras una determinada situación familiar, aunque la creciente complejidad de dichas trayectorias augura este uso cada vez menos pertinente. El censo de población sobreestima, en relación con las otras fuentes de información, el número de monoparentales casadas. Véase, en el gráfico 7, la diferencia del peso de las casadas en los núcleos monoparentales en el censo de población en relación con el que se muestra en las otras dos fuentes (las EPAs y la reciente Enquesta demogràfica 2007 referida a la población residente en Cataluña). Treviño (2011) sostiene que el hecho de que la última operación censal se realizase a la par que la renovación padronal puede haber influido en esta sobrestimación de núcleos monoparentales de población casada ${ }^{6}$. La coherencia de las fuentes en relación con el estado civil de las madres de núcleos reconstituidos es, en cambio, total.

6. Dada la naturaleza administrativa del padrón de habitantes y su función acreditativa de la residencia, la simultaneidad de la operación censal y padronal en el año 2001 (incluso los datos padronales estaban preimpresos en el cuestionario censal), podría haber conducido, en algunos casos, a algunas personas a la falsa idea de que podría serles beneficioso tergiversar la declaración de parentesco o de residencia de la madre y de los hijos en función de unos intereses personales determinados (como ocurre con el padrón, por ejemplo, para la obtención de plazas en determinados colegios o guarderías) o a no corregir los datos padronales preimpresos en el censo de población. 
Mientras el 94\% de las madres que forman núcleos biparentales no reconstituidos son casadas, sólo lo son el 46\% de las reconstituidas. Un 30\% de éstas últimas son separadas o divorciadas y un $17 \%$, solteras. Entre un período y otro, se incrementa el peso de las separadas y divorciadas, así como el de las solteras. Esto es reflejo del peso de las parejas de hecho entre los núcleos reconstituidos. En efecto, más de la mitad de las madres de estos núcleos reconstituidos están en una unión consensual ${ }^{7}$, mientras que sólo lo están el 6\% de las madres de núcleos no reconstituidos con algún hijo menor de 29 años. Esta tendencia a la consensualidad muestra una evolución al alza (en el primer periodo, las parejas de hecho representan un $44 \%$ de los núcleos reconstituidos). Este rasgo es uno de los más destacados en la bibliografía (Bumpass et al., 1990; Stewart, 2001; Cherlin, 1978 y 2004) para apoyar la hipótesis de la reconstitución como una institución incompleta que sirve para dar cuenta de la baja prescripción normativa de la configuración de los roles de parentesco en estas familias.

\section{La nacionalidad}

El impacto de la inmigración extranjera en la diversificación de las estructuras familiares en España es un hecho constatado a partir de la intensificación de los flujos inmigratorios producida en el primer quinquenio del año 2000. En el gráfico 8 , puede observarse que ese impacto es más intenso entre las mujeres que viven en las estructuras familiares objeto de estudio que entre las que forman núcleos biparentales no reconstituidos. Este elevado peso de los núcleos monoparentales en la estructura familiar de los extranjeros ha sido destacado como un aspecto que tiene más que ver con las estrategias migratorias predominantes entre los ciudadanos de cada una de las nacionalidades que con la disolución de la pareja (Domingo y Bayona, 2010). No obstante, si se calcula el indicador transversal de reconstitución, es decir, el número de mujeres en núcleos reconstituidos por 100 monoparentales, la relación se dobla entre las mujeres de nacionalidad extranjera en el segundo período (40 versus 19 entre las de nacionalidad española) y es aún más intensa en el primer periodo (41 versus 15 con datos de la EPA 1999-2004 y de 37 versus 15 con datos del censo de 2001). No es descabellado plantear que, en un contexto migratorio, las perspectivas de una separación conyugal larga pueden evitar el disfrute de los beneficios del matrimonio y alentar la formación de familias reconstituidas para obtenerlos.

\section{La participación laboral}

Los niveles de participación laboral entre las madres monoparentales y reconstituidas son muy altos (gráfico 9), pero son superiores entre las primeras en todas

7. Este porcentaje podría incluso ser algo más elevado teniendo en cuenta que se han estimado como uniones consensuales todas aquellas que no correspondiesen a las de una pareja cuyos dos miembros registrasen estado civil de casado, pero lo cierto es que, en la EPA, no se especifica si las uniones de dos personas con estado civil de casado corresponden a las de una pareja casada entre sí. 
Gráfico 8. Porcentajes de madres de nacionalidad no española sobre el total según tipo de núcleo familiar (diversas fuentes y periodos)

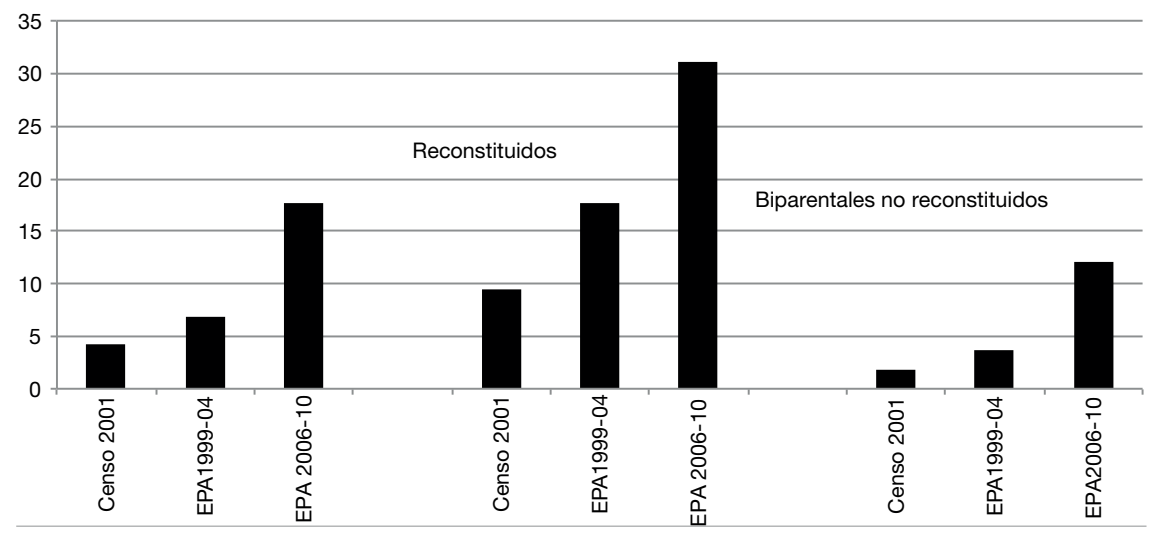

Núcleos con hijos menores de 29 años.

Fuente: elaboración propia a partir de los microdatos del censo y de la EPA.

Gráfico 8. Tasas de actividad de las mujeres de los núcleos reconstituidos en los que ella aporta a los hijos comunes y de los núcleos monoparentales femeninos (años 1999-2004 y 2005-2010)

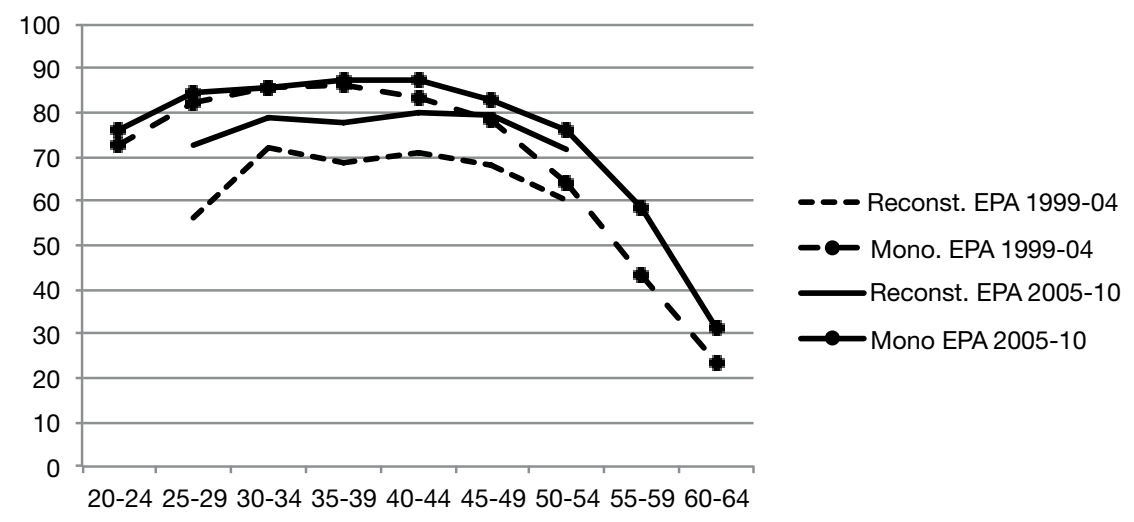

Núcleos con hijos menores de 29 años.

Fuente: elaboración propia a partir de los microdatos de la EPA.

las edades. Este perfil de menor actividad laboral de las madres reconstituidas en relación con las monoparentales reforzaría la tesis de que la reconstitución selecciona a las más necesitadas como una salida para mejorar el estatus económico de la familia. No obstante, entre un periodo y otro, las diferencias de actividad de las madres se reducen mucho, al incrementarse, sobre todo, la participación entre las jóvenes madres de núcleos reconstituidos. Los datos censales confirman esta diferencia de perfiles, si bien subestimando los niveles de actividad de todas las madres. De todas maneras, hay que recordar que 
estamos ante una descripción transversal y que la relación entre los cambios de situación de actividad y situación familiar es compleja. Un estudio anterior (Treviño, 2011) ha mostrado, por ejemplo, que la entrada de las mujeres en situación de monoparentalidad tiene un doble efecto: selecciona a las mujeres que estaban ocupadas en el momento de entrar en monoparentalidad, pero además, alienta la ocupación de las mujeres que entraron en monoparentalidad sin estar ocupadas.

\section{De la monoparentalidad a la reconstitución familiar: análisis multivariable}

A falta de datos longitudinales, se analiza la cuestión del perfil sociodemográfico selectivo de las mujeres que pasan de una situación de monoparentalidad a la reconstitución a través de fuentes estadísticas transversales. Para ello, se selecciona en cada momento el conjunto de mujeres que alguna vez han estado en situación de monoparentalidad (las que, en el momento del censo y de la encuesta, encabezan un núcleo monoparental o que forman parte de un núcleo reconstituido en el que ellas aportan a los hijos no comunes) y se configura para los modelos de análisis multivariable por regresión logística una variable dicotómica, en la que las mujeres que están en un núcleo reconstituido asumen el valor de $1 \mathrm{y}$, el resto, las monoparentales, el valor de 0 . Las covariables introducidas responden a las variables clave que la literatura destaca como esenciales para determinar la suerte de una nueva unión o la salida de una situación de monoparentalidad (Bumpass et al., 1990; McCarthy y Cherlin, 1990; Ermisch y Wrigth, 1991; Desrosiers y Le Bourdais, 1993; Le Bourdais et al., 1995; Juby et al., 2001; De Graaf y Kalmijn, 2003): la edad y el estatus de la mujer (nivel educativo y relación de actividad), el número de hijos y la edad que tienen. Se ha introducido, así mismo, la variable nacionalidad, aun sabiendo que la unión matrimonial facilita la adquisición de la nacionalidad española ${ }^{8}$, ya que es una de las variables básicas a distinguir en los estudios de comportamiento familiar. Dado que se pretende analizar la cuestión de la selectividad de madres monoparentales que entran en una familia reconstituida, la variable de número de hijos hace referencia a los hijos propios, es decir, en las mujeres de núcleos reconstituidos se refiere exclusivamente a los hijos que ellas han aportado al núcleo familiar. Esta distinción es importante, ya que, como se ha visto en el análisis descriptivo, el sello institucionalizador del núcleo familiar reconstituido mediante un hijo común es relativamente frecuente en España. De este modo, la asociación que encontremos entre cada una de las variables, aunque no puede interpretarse en términos de causalidad, sí que está estandarizada por el efecto conjunto de las variables introducidas.

En primer lugar, cabe destacar la coherencia de las dos fuentes utilizadas en los resultados. Tanto en el modelo realizado con los datos censales como en el

8. Basta un año de residencia en España para aquél que, en el momento de la solicitud, lleve un año casado (Art. 22.2 del Código Civil). 
de la EPA del primer periodo, el procedimiento de introducción de las variables paso a paso, utilizado con el fin de identificar a las variables con mayor capacidad explicativa, señala la edad de las mujeres, el número de hijos y la relación de actividad, por este orden, como esas variables. En el último período, la nacionalidad pasa a ser la tercera variable de mayor poder explicativo, y la relación de actividad se sitúa por detrás.

Se confirma uno de los resultados más concluyentes y resaltados en la literatura: la relación negativa de la reconstitución con la edad pero con matices (tabla 1). La propensión de las mujeres de 50 y más años a formar un núcleo reconstituido es un $67 \%$ más baja que la de las mujeres de menos de 34 años. No obstante, las que tienen entre 35 y 49 años presentan una propensión algo más elevada que las muy jóvenes? . Dado el calendario tan tardío de la formación familiar en España, las mujeres que han pasado por una situación de monoparentalidad y tienen menos de 34 años inscriben su biografía familiar en un contexto realmente excepcional. Los ya citados estudios de monoparentalidad en España destacan que la frecuencia de las madres monoparentales de corresidir con los progenitores es muy elevada, especialmente entre las muy jóvenes. Podría plantearse como hipótesis que, entre ellas, este sesgo no controlado explique la menor asociación que muestran con la reconstitución.

La nacionalidad no española también presenta un efecto positivo con la reconstitución familiar. El alejamiento del país de origen parece favorecerla, pero la variable acerca de la nacionalidad no española agrega un conjunto de nacionalidades con comportamientos heterogéneos que hacen difícil una única interpretación.

El hecho de tener más de un hijo, atendiendo al efecto del conjunto de variables introducidas en el modelo, disminuye a la mitad la asociación con la reconstitución de las mujeres alguna vez en situación de monoparentalidad. Este efecto se mantiene en todos los periodos y se percibe con ambas fuentes. Este resultado se alinea con el obtenido en algunos estudios realizados en demografía desde la perspectiva del mercado matrimonial (Lundberg, 2006). Estos estudios argumentan que el valor del matrimonio para las madres monoparentales depende de lo que mejore su situación con el matrimonio, hecho que, a su vez, está ligado a las características de los futuros maridos o parejas, que, igualmente, están conectadas con su propio atractivo en este mercado para poder encontrar a ese marido adecuado. Para el futuro marido, los hijos de estas madres afectan a sus perspectivas en recursos de dinero y de tiempo con la madre. Para la mujer, los resultados son más ambiguos, porque, por un lado, la situación económica de las madres monoparentales es más precaria (Eurostat, 2010) y, por tanto, la maternidad puede aumentar la demanda de un cónyuge que proporcione recursos adicionales, pero, por otro lado, su menor valor en el mercado matrimonial puede dificultar encontrar ese esposo

9. La variable correspondiente a la edad se ha introducido en forma categórica, porque es como mejor se recoge el comportamiento de la variable, que no se ajusta ni de manera continua, ni cuadrática, ni logarítmica. 
Tabla 1. Determinantes de la reconstitución familiar. Mujeres que alguna vez han vivido en situación de monoparentalidad (mujeres que encabezan núcleos monoparentales y que viven en núcleos reconstituidos con hijos propios) (diversas fuentes y periodos)

\begin{tabular}{|c|c|c|c|}
\hline & $\begin{array}{l}\text { Censo } 2001 \\
\quad \operatorname{Exp}(B)\end{array}$ & $\begin{array}{l}\text { EPAs 1999-2004 } \\
\quad \operatorname{Exp(B)}\end{array}$ & $\begin{array}{c}\text { EPAs } 2005-20010 \\
\operatorname{Exp(B)}\end{array}$ \\
\hline \multicolumn{4}{|l|}{ Edad } \\
\hline$<34$ & 1 & 1 & \\
\hline $35-49$ & $1,2^{\star \star \star}$ & $1,2^{\star \star \star}$ & $1,2^{\star \star \star}$ \\
\hline $50+$ & $0,3^{\star \star \star}$ & $0,2^{\star \star \star}$ & $0,4^{\star \star \star}$ \\
\hline \multicolumn{4}{|l|}{ Nacionalidad } \\
\hline Española & 1 & 1 & 1 \\
\hline No española & $2,0^{\star \star \star}$ & $2,2^{\star \star \star}$ & $1,8^{\star \star \star}$ \\
\hline \multicolumn{4}{|l|}{ Relación de actividad } \\
\hline Ocupados & 1 & 1 & 1 \\
\hline Parados & $1,0 \mathrm{NS}$ & $0,9^{\star}$ & $1,0 \mathrm{NS}$ \\
\hline Inactivos & $1,5^{\star \star \star}$ & $1,9^{\star \star \star}$ & $1,4^{\star \star \star}$ \\
\hline \multicolumn{4}{|l|}{ Nivel de instrucción } \\
\hline Obligatorios & 1 & 1 & 1 \\
\hline Segundo nivel & $0,9^{\star \star \star}$ & $1,0 \mathrm{NS}$ & $1,1 \mathrm{NS}$ \\
\hline Tercer nivel & $0,6^{\star \star \star}$ & $1,1 \mathrm{NS}$ & $0,9^{\star \star}$ \\
\hline \multicolumn{4}{|l|}{ Número de hijos } \\
\hline Un hijo & 1 & 1 & 1 \\
\hline Dos o más hijos & $0,5^{\star \star \star}$ & $0,4^{* \star *}$ & $0,5^{\star \star \star}$ \\
\hline Constante & $0,2^{\star \star *}$ & $0,2^{\star \star \star}$ & $0,3^{\star \star \star}$ \\
\hline -2 Log likelihood & 40201 & 3920000 & 6340000 \\
\hline Cox \& Snell R Square & 0,049 & 0,082 & 0,054 \\
\hline Nagelkerke R Square & 0,088 & 0,15 & 0,088 \\
\hline $\mathrm{N}$ casos & 52709 & 23061 & 25048 \\
\hline
\end{tabular}

Núcleos con hijos menores de 29 años.

Fuente: elaboración propia a partir de los microdatos del censo y de la EPA.

adecuado. Aunque no podamos analizarlo, las pocas evidencias empíricas sobre el efecto de los hijos en las probabilidades de unión de los hombres señalan que, tanto si se trata de hijos corresidentes como si no, pero sobre todo si lo son, éstos incrementan la suerte de una nueva unión. Dada la diferencia de la presencia de los hijos en la vida diaria de los hombres y de las mujeres y de sus cónyuges, estas diferencias son claves para explicar los roles de género en las pautas de formación de la pareja (Goldscheider y Sassler, 2006).

Las madres inactivas tienen una asociación con la reconstitución que dobla al de las madres ocupadas en el primer periodo y que es un $40 \%$ mayor en el segundo. Esta relación refuerza la hipótesis de la reconstitución como una estrategia de mejora económica del núcleo, sobre todo teniendo en cuenta que la monoparentalidad se asocia a un mayor riesgo de pobreza. Ahora bien, entre un periodo y otro, parece disminuir la fuerza de esta variable. Está disminución es de difícil interpretación. En la línea argumentada 
por Blossfed et al. (1995), la explicación podría apoyarse en su hipótesis de que las variables de estatus individual tienen mayor impacto en explicar los comportamientos sociales emergentes y pioneros, y se observa una relación positiva entre estatus individual y ese comportamiento, relación que pierde su fuerza a medida que se legitima, se normaliza y se difunde un comportamiento social determinado.

De hecho, la otra variable de estatus de las mujeres, el nivel de instrucción, señala unos parámetros que podrían interpretarse en la misma línea. Las diferencias parecen marcarse entre las mujeres de menor y de mayor nivel educativo. Entre las universitarias, el censo de población muestra una asociación con la reconstitución un $40 \%$ menor respecto a las que no tienen estudios o sólo han cursado los obligatorios. Y sólo las EPAs del segundo periodo muestran una asociación significativa en la misma dirección, aunque de mucha menor magnitud (un 10\% menor de las universitarias respecto a las de más bajo nivel de instrucción).

\section{Reflexiones finales}

La dificultad de disponer de fuentes estadísticas pertinentes para analizar la complejidad del perfil sociodemográfico de las familias reconstituidas desde una perspectiva cuantitativa es una situación que fue denunciada casi dos décadas atrás en otros países de nuestro entorno (Duchêne, 1990) y que, en España, continúa siendo una realidad que, además, no tiene visos de cambiar a corto plazo. La diversidad tipológica de las familias reconstituidas y su naturaleza esencialmente dinámica exigirán recoger información detallada sobre el historial de pareja y de fecundidad, no sólo de los individuos encuestados, sino también de sus cónyuges.

Hoy, aunque los datos nos señalen en España un porcentaje de custodia compartida muy bajo entre las parejas que se divorcian, éste está en aumento y hace prever un mayor crecimiento, no sólo porque la última reforma de la ley de divorcio refuerza su figura, sino, también, porque la mayor incorporación de las mujeres al mercado de trabajo la alienta. En este contexto, los estudios transversales de la reconstitución familiar no serán en ningún modo pertinentes. Las fuentes estadísticas tendrán que resolver el problema de la datación de la convivencia de los sujetos entrevistados con los diferentes tipos de hijos (los propios y los de sus parejas). Por otro lado, los estudios cualitativos (Solsona, 2011) ya nos anuncian que el abordaje del estudio sociodemográfico de las familias reconstituidas reclamará también información sobre la naturaleza de las relaciones paternofiliales y maternofiliales, en concreto, sobre la calidad de esas relaciones en términos de intensidad, continuidad y dimensiones de los contactos (económicas, de cuidado, etc.) para poder resignificar el rol de la maternidad y de la paternidad en estos nuevos contextos de reconstitución familiar.

En este trabajo, se ha intentado sortear las dificultades de las fuentes estadísticas para aportar una primera y modesta contribución al estudio 
de la reconstitución familiar usando una perspectiva de análisis transversal con los datos que ofrece la EPA, que, hoy por hoy, se está convirtiendo no sólo en la fuente por antonomasia para el estudio de las características de la fuerza de trabajo, sino en una fuente forzosamente cada vez más utilizada en el campo de los estudios sociológicos de la familia. La información actualizada que ofrece la EPA y la coherencia mostrada en el perfil sociodemográfico de los núcleos reconstituidos en relación con el censo avala la pertinencia de su uso.

Los resultados del perfil de madres monoparentales que se asocia con una mayor reconstitución familiar se alinean con los obtenidos en otros países de nuestro entorno desde perspectivas biográficas, y ello refleja las dinámicas de género de los mercados matrimoniales secundarios. No obstante, no pueden de ningún modo suplir el estudio dinámico de la reconstitución familiar. Las variables de estatus individual de las mujeres y, en especial, la relación de actividad, tienen una relación negativa con la reconstitución familiar, pero parecen perder fuerza entre los dos periodos estudiados. Este resultado es sorprendente y lo hemos interpretado en los términos de Blossfed et al. (1995) como un posible efecto de la difusión y la legitimación de comportamientos sociales en un principio emergentes, si bien necesita ser explorado con más detenimiento y basándose en encuestas biográficas. Son la edad de la madre y el número de hijos las variables con mayor poder predictivo y, en el último período, también la nacionalidad. Ser una madre monoparental joven, tener menos hijos y no poseer la nacionalidad española se asocia con una mayor propensión a la reconstitución.

El rol de los hijos en las pautas de reconstitución familiar necesita también mayor atención analítica. El incremento del divorcio conlleva rápidos cambios en el mercado matrimonial con el incremento de la presencia de individuos previamente unidos y con hijos. La reconstitución implica, por tanto, la configuración de nuevas figuras maternas y paternas. Ello complica la dinámica de género en la elección de la pareja y en el funcionamiento de los mercados matrimoniales. En este sentido, será imprescindible analizar el papel de los hijos propios corresidentes y no corresidentes y el de los hijos de los futuros cónyuges en la determinación de las dinámicas de género de la reconstitución familiar. Las fuentes analizadas no nos han permitido analizar ni el perfil de la reconstitución familiar de los hombres ni el papel de los hijos no corresidentes de las mujeres y de sus parejas en las pautas de unión de las madres monoparentales.

Finalmente, resta decir que el perfil sociodemográfico de las madres monoparentales es diferente en el momento de entrada y en el momento de salida de la situación de monoparentalidad (momento de entrada en un núcleo reconstituido), por ejemplo: la relación de actividad, porque el estatus de monoparentalidad selecciona a las ocupadas y, además, alienta la ocupación (Treviño, 2011). Esto implica que la duración de la monoparentalidad puede tener un impacto importante en determinar la suerte de la reconstitución que es necesario controlar. El ejercicio de análisis realizado no tiene 
presente el tiempo de monoparentalidad ni de reconstitución que llevan las madres que se comparan. Algunos estudios cualitativos muestran que las dificultades que experimentan las familias monoparentales en el momento de formación se ven aliviadas por un proceso de reacomodación a la nueva situación que implica cambios en diferentes facetas de sus vidas, y ello puede afectar a las oportunidades y a las expectativas en torno a la reconstitución familiar (Solsona, 2011).

\section{Referencias bibliográficas}

Blossfeld, H.-P.; De Rose, A.; Hoem, J. M. y Rohwer, G. (1995). «Education, modernization, and the risk of marriage disruption in Sweden, West Germany, and Italy». En: OpPenHeim, K. y Jensen, A.-M. (eds.). Gender and Family Change in Industrialized Societies. Oxford: Clarendon Press, 200-222.

BumPASS, Larry; SWEET, James y CASTRO MARTín, Teresa (1990). «Changing Patterns of Remarriage». Journal of Marriage and the Family, 52 (3), 747-756.

CASTANYS, Elisenda (2006). «Familias reconstituidas y adolescencia». Aula de Innovación Educativa, 149, 19-23.

Chambaz, Christine (2001). «Lone-parent Families in Europe: A Variety of Economic and Social Circumstances». Social Policy, 35 (6), 658-671.

CHERLIN, Andrew (1978). «Remarriage as an incomplete institution». Journal of Family Issues, 84, 634-650.

- (2004). «Deinstitutionalization of American Marriage». Journal of Marriage and Family, 66 (4), noviembre, 848-861.

COLEMAN, Marilyn; GANONG, Lawrence y FINE, Mark (2000). «Reinvestigating remarriage: Another decade of progress". Journal of Marriage and the Family, 62, 12881307.

De GraAf, Paul M. y Kalmijn, Matthijs (2003). «Alternative Routes in the Remarriage Market: Competing-Risk Analyses of Union Formation after Divorce». Social Forces, 81 (4), 1459-1496.

Desrosiers, Hélène y Le Bourdais, Céline (1993). «La Dynamique de la Monoparentalité Fémenine au Canada». European Journal of Population, 9, 197-224.

DOMINGO, Andreu y BAYONA, Jordi (2010). «Los hogares de la población de nacionalidad extranjera en España en el año 2001». Papers: Revista de Sociología, 95 (3), 731-754.

DuCHÊNE, J. (1990). «Les familles monoparentales et recomposées. Quelles données pour une mesure de leur incidence dans les pays développés: permanences et changements». En: PRIOUX, F. (dir.) La famille dans les pays développés: permanences et changements. París: INED. Congrès et Colloques, 25-41.

EDIN, Kathryn (2000). «What Do Low-Income Single Mothers Say about Marriage?». Social Problems, 47 (1), 112-133.

ERMISCH, John y WrighT, Robert (1991). «The duration of Lone Parenthood in Great Britain». European Journal of Population, 7, 129-158.

EUROSTAT (2010). Combating poverty and social exclusion: A statistical portrait of the European Union 2010. Luxemburgo: Publications Office of the European Union.

FLAQUER, Lluís y SOLER, Joan (1990). Permanencia y cambio en la familia española. Madrid: Centro de Investigaciones Sociológicas. 
García-Mina Freire, Ana; Carrasco Galán, Maria José; Espinar Fellmann, Isabel; MARTínez DíAz, María Pilar (2003). «Familias reconstituidas: un estudio sobre las nuevas estructuras familiares». Clínica y Salud: Revista de Psicología Clinica y Salud, 14 (3), 301-33.

GARRIDO, Luís; Requena, Miguel y TOHARIA, Luis (2000). «La encuesta de población activa desde la perspectiva de los hogares». Estadística Española, 42 (146), 115-155.

GOldSCHEIDER, F. y SASSLER, S. (2006). "Creanting stepfamilies: Integrating Children into the Study of Union Formation». Journal of Marriage and the Family, $68,275-291$.

Guilarte Martín-Calero, Cristina (2009). «El ejercicio de la patria potestad en situaciones de ruptura convivencial: Análisis jurisprudencial y propuesta de reforma del Código Civil». En: Guilarte Martín-Calero, Cristina (coord.). Aspectos civiles y penales de las crisis matrimoniales. Valladolid: Lex Nova.

Juby, Heritier; Marcil-Gratton, Nicole y Le Bourdais, Celine (2001). «A Step Further in Family Life: The Emergence of the Blended Family». En: BÉLANGER, Alain (ed.). Report on the Demographic Situation in Canada 2000. Otawa: Statistics Canada.

JurAdO, Teresa (2005). «Las nuevas familias españolas». En: GONZÁLEZ, Juan Jesús y ReQuena, Miguel (eds.). Tres décadas de cambio social en España. Madrid: Alianza Editorial, 51-77.

Le Bourdais, Céline; Desrosiers, Hélène y Laplante, Benoît (1995). «Factors Related to Union Formation Among Single Mothers in Canada». Journal of Marriage and the Family, 57, 410-420.

LundberG, S. y Rose, E. (2006). "Child gender and the transition to marriage». Demography May, 40 (2), 333-49.

MCCARTHY, James y CHERLIN, Andrew (1990). «Caractéristiques demográphiques des familles monoparentales et des familles recomposés». En: PriOuX, France (ed.). La famille dans les pays développés: Permanences et changements. París: INED, UIESP, CNAF, CNRS, 131-144.

MotT, Frank L. y Moore, Sylvia F. (1983). «The Tempo of Remarriage Among Young American Women». Journal of Marriage and the Family, 45 (2), 427-436.

Prskawetz, Alexia; Vikar, Andrés; Philipov, Dimitier y EngelHardT, Henriette (2003). «Pathways to Stepfamily Formation In Europe: Results from the FFS». Demographic Research, 8 (5), 107-149.

Rendall, Michael S. (1999). «Entry or Exit? A Transition-Probability Approach to Explaing the High Prevalence of Single Motherhood Among Black Women». Demography, 36 (6), 369-376.

REQUenA, Miguel (1999). «Pautas contemporáneas de evolución de los hogares en España». Revista Internacional de Sociología, 22, 63-65.

RIVAS RIVAS, Ana María (2008). «Las nuevas formas de vivir en familia: El caso de las familias reconstituidas». Quaderns de Relacions Laborals, 26 (1), 179-202.

Roigé VenturA, Xavier (2006). «Las familias mosaico: Recomposición familiar tras el divorcio». En: Roigé Ventura, Xavier (coord.). Familias de ayer, familias de hoy. Barcelona: Icaria.

SOlSONA, Montserrat (2011). "(Re) Construcció de les maternitats i de les paternitats després d'un divorci. Notes d'un estudi qualitatiu». Treballs de la Societat Catalana de Geografia, 69, 141-160.

Solsona, Montserrat y Treviño, Rocío (1990). Estructuras familiares en España. Madrid: Ministerio de Asuntos Sociales. Instituto de la Mujer. 
STEWART, Susan D. (2001). "Contemporary American stepparenthood: Integrating cohabiting and nonresident stepparents». Population Research and Policy Review, 20, 345-364.

TREVIÑo, Rocío (2011). La monoparentalidad en la encrucijada, perfiles y dinámica. Madrid: Editorial Académica Española.

Treviño, Rocío; GumÀ, Jordi; Permanyer, Iñaqui (en prensa). Nuevas familias e igualdad de género: las familias reconstituidas.

VALERO, Ángeles (1995). «El sistema familiar español recorrido a través del último siglo». Reis, 70, 91-105. 